\title{
Casseroles, légumes et halloween
}

Une approche multiscalaire des phénomènes de diffusion

Multiscalar Approachs of Diffusion Phenomena

Renaud Zeebroek, Jean-Michel Decroly et Olivier Gosselain

\section{(2) OpenEdition}

Journals

Édition électronique

URL : https://journals.openedition.org/tc/4586

DOI : $10.4000 /$ tc. 4586

ISSN : 1952-420X

Éditeur

Éditions de l'EHESS

Édition imprimée

Date de publication : 15 août 2009

Pagination : 50-73

ISBN : 978-2-7351-1235-7

ISSN : 0248-6016

\section{Référence électronique}

Renaud Zeebroek, Jean-Michel Decroly et Olivier Gosselain, "Casseroles, légumes et halloween »,

Techniques \& Culture [En ligne], 51 | 2009, mis en ligne le 15 juin 2011, consulté le 29 septembre 2022.

URL : http://journals.openedition.org/tc/4586 ; DOI : https://doi.org/10.4000/tc.4586 


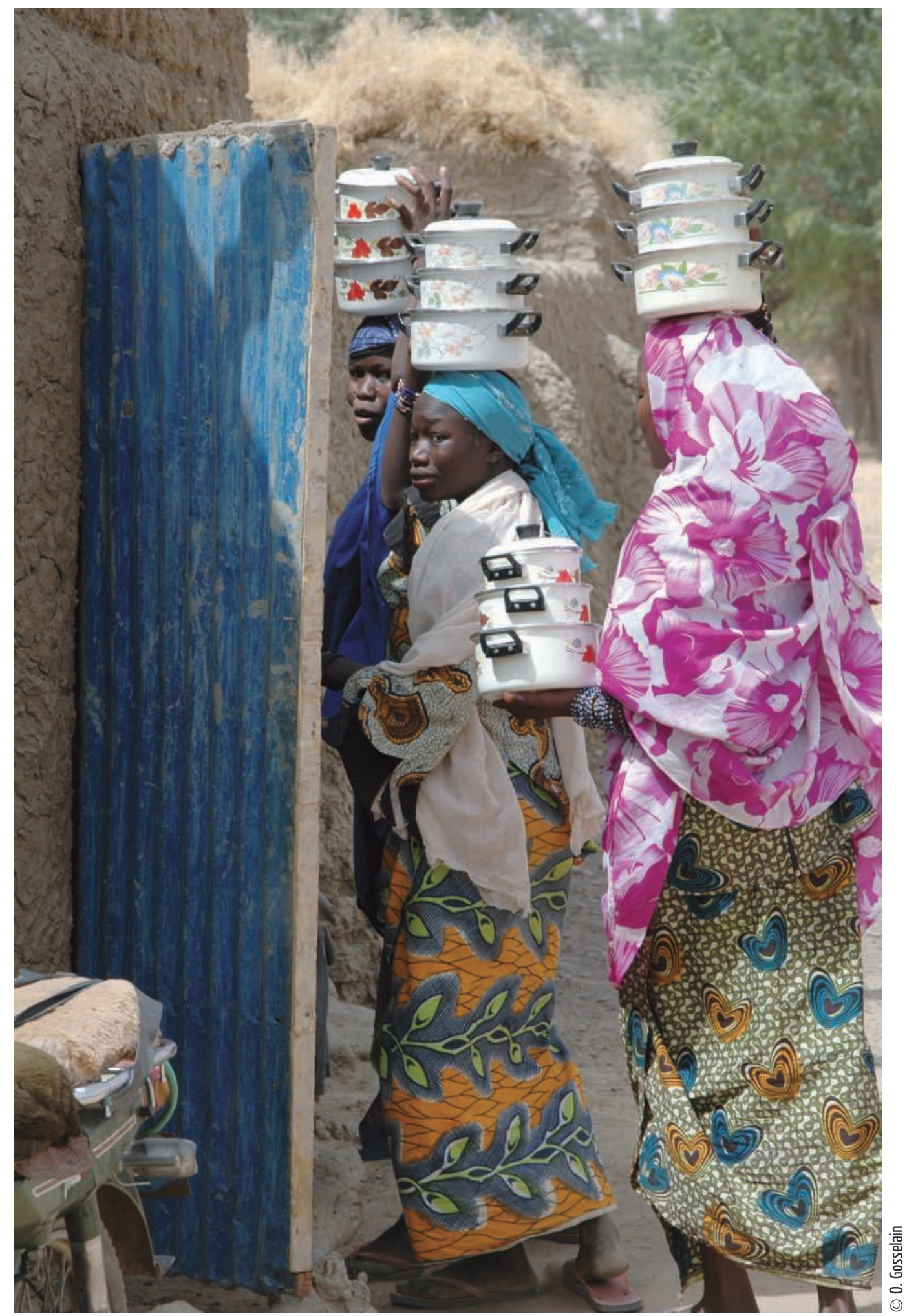


Renaud Zeebroek, Jean-Michel Decroly

\& Olivier Gosselain

Des choses, des gestes, des mots

Université libre de Bruxelles

Techniques \& Culture 51, 2008 : 50-73

\title{
CASSEROLES, LÉGUMES ET HALLOWEEN
}

\section{Une approche multiscalaire des phénomènes de diffusion}

\author{
Où l'on revient sur la trajectoire improbable des produits émaillés au Niger et sur \\ les autres études de cas présentées dans ce numéro, afin de proposer un cadre \\ d'analyse plus général des phénomènes de diffusion.
}

Dans l'article précédent (Gosselain et al., ce volume), nous avons retracé la trajectoire d'une catégorie particulière de produits - récipients émaillés - au sein des trousseaux de mariage de populations du Sud Niger. Cette étude a fait apparaître un double phénomène de diffusion, celui des objets et celui de leurs usages. Il s'agit maintenant d'en comprendre les logiques dans une perspective plus générale.

La question de la diffusion des traits culturels est presque aussi vieille que l'anthropologie. Rappelons que son importance a été reconnue au tournant du XX $\mathrm{XX}^{\mathrm{e}}$ siècle, aussi bien en Allemagne (Kulturkreise), qu'aux États-Unis (École de Franz Boas) ou encore en France, avec un des fondateurs de la géographie humaine, Paul Vidal de La Blache. Par la suite, cette thématique a également irrigué les études portant sur « l'acculturation », avec les travaux de Melville Herskovits aux États-Unis ou ceux de Roger Bastide en France. Depuis la fin des années 1960 et à quelques exceptions près, les chercheurs qui s'intéressent encore aux phénomènes de diffusion - c'est-à-dire à la trajectoire à la fois spatiale, sociale et historique des traits culturels - s'inspirent principalement de la théorie de 


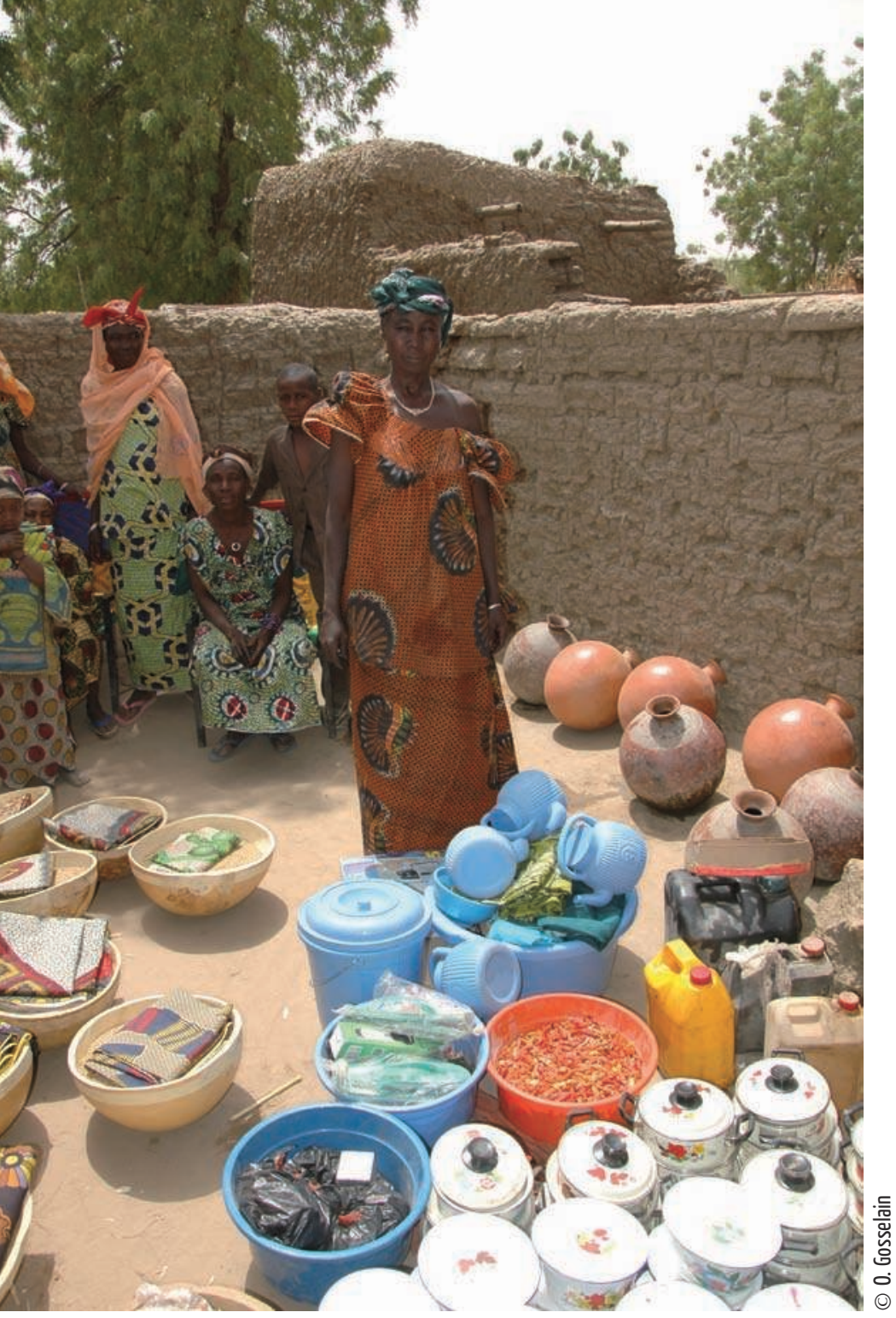

la diffusion des innovations, élaborée par Everett Rogers dès 1962 et plusieurs fois reformulée (Rogers 1995). Cette théorie se révèle pourtant peu satisfaisante à bien des égards. Pour le dire rapidement, la question des canaux d'introduction et d'approvisionnement de la nouveauté n'y est pas abordée, le rôle de filtre du contexte social et culturel y est reconnu, mais ses modalités d'action ne sont pas étudiées, de sorte que les transformations de la nouveauté, concomitantes à son adoption, restent dans l'ombre. Enfin, la théorie de la diffusion des innovations prévoit bien la possibilité du rejet d'une nouveauté, mais n'en dit rien qui nous permettrait de comprendre, par exemple, le comportement singulier des Peuls et des Touaregs détaillé dans l'article précédent (Gosselain et al., ce volume).

Les zones d'ombre de la théorie de Rogers affectent particulièrement ces moments clés qui vont de l'introduction d'une nouveauté à son adoption. Ils forment en quelque sorte le revers d'une théorie qui s'est surtout consacrée aux conditions de propagation des innovations. L'essentiel des modélisations développées par Rogers vise en effet à expliquer le rythme et l'ampleur de l'adoption, considérée comme une variable dépendante. Foncièrement utilitariste, un tel modèle se focalise sur les diffusions incitées et sur les moyens les plus efficaces de les mener à

\section{Mariage à Waraou (Niger)}

Dans la concession de la jeune mariée, où sont rassemblés parents et amis, la mère offre à la vue de tous les produits qu'elle a contribué à accumuler depuis plusieurs mois et qui seront transportés le jour même vers la concession de l'époux.

(Fig. 1) bien, au détriment d'une compréhension des processus qui gouvernent les diffusions spontanées. Notre objectif est au contraire de proposer un modèle d'analyse dédié à la compréhension des phénomènes de diffusion, plutôt qu'à leur manipulation.

L'inflexion particulière de la théorie de Rogers provient de ses origines disciplinaires. Elle s'est en effet d'abord développée en sociologie rurale, autour de l'action d'organismes publics. Il s'agissait alors d'encourager la diffusion d'innovations techniques, qui étaient considérées par leurs promoteurs comme intrinsèquement meilleures que ce qui leur préexistait. Si un certain nombre des naïvetés d'origine ont été corrigées au fil du temps et des nombreuses études de cas (Rogers 1995), l'inflexion propagatrice n’a jamais été remise en cause. C'est ce qui explique le succès de cette théorie dans différents domaines comme la sociologie rurale, la santé publique, le système éducatif, la communication et surtout le marketing. Inversement, on remarquera que, de l'avis même de Rogers (1995, table 
2.1), l'anthropologie sociale et la géographie humaine ont peu contribué au modèle. Ce relatif silence provient d'un intérêt ancien de nos disciplines pour les conditions d'introduction et de réception d'une nouveauté, plutôt que pour les manières d'assurer son succès.

Dans la perspective utilitariste de Rogers, la nouveauté est intrinsèquement avantageuse, ce qui dispense de chercher ailleurs les raisons de son adoption (Rogers 1995: 109, 241). Il en résulte un relatif désintérêt pour le système social où diffuse la nouveauté, ainsi que pour les éventuels processus d'adaptation (ce que Rogers nomme la réinvention). Par ailleurs, Rogers considère l'adoption d'une nouveauté comme un processus fondamentalement individuel. Cet accent mis sur l'individu le conduit logiquement à se focaliser sur les leaders d'opinion, les agents du changement et les réseaux.

La définition qu'il propose du « système social » est cohérente avec ce point de vue: il s'agit d'un « ensemble d'unités en relation, engagées dans la résolution commune d'un problème en vue d'atteindre un même objectif » (1995 : 23). Cette vue par le petit bout de la lorgnette lui permet de présenter un groupe de fermiers de l'Iowa comme un système social à l'intérieur duquel l'usage d'un pesticide diffuse. On remarquera au passage que la définition de Rogers évoque fortement celle de « communauté de pratique » élaborée par Lave et Wenger (1991; Wenger 1998), qui offrent une grille de lecture beaucoup plus performante, sans prétendre pour autant que les groupes qu'ils étudient sont représentatifs de systèmes sociaux. Quoi qu'il en soit, l'échelle privilégiée par Rogers ne permet pas d'analyser des phénomènes de propagation dans des groupes sociaux de grande envergure, comme c'est le cas des populations qui utilisent aujourd'hui des produits émaillés dans un cadre matrimonial.

Cette vue simplifiée du système social a été partiellement corrigée dans d'autres champs de recherche, particulièrement ceux du marketing et des études relatives au comportement du consommateur (par exemple Gatignon \& Robertson 1985). De tels travaux se focalisent sur les diffusions de masse par l'intermédiaire du marché, où les objets proposés doivent rencontrer les désirs, les attentes du consommateur. Ici, les chercheurs s'intéressent bien plus aux effets des normes et des valeurs propres au contexte culturel, ainsi qu'aux raisons qui expliquent le succès ou le rejet d'un objet particulier. Leurs études rejoignent une approche plus anthropologique, en soulignant que « l'avantage relatif » est une notion socialement construite et que l'adoption d'une nouveauté est un phénomène collectif (Arnould 1989). Par contre, elles attribuent un rôle fondamental à la recherche de la différenciation sociale, facteur qui, à lui seul, ne peut rendre compte de la dynamique engendrée par la rencontre entre une nouveauté et un système social (voir notamment Miller 1995). De même, reconnaître le rôle favorable, ou non, du « milieu technique » dans la réception d'une innovation n'aboutit qu'à créer une nouvelle « boîte noire » si l'on néglige d'identifier précisément les aspects du « milieu » qui sont à l'œuvre (voir illustrations et discussion dans Leroi-Gourhan 1992 [1945] ; Creswell 1983; Edgerton 1998). 
Il est surprenant de constater que si Rogers reconnaît explicitement que cette rencontre est décisive pour le futur de la nouveauté et que sa « réinvention » fait partie intégrante de ce processus (1995 : 179-180), il n'en modifie pas pour autant sa perspective d'analyse. Tout se passe comme s'il ne s'agissait que de facteurs complémentaires à prendre en compte pour pouvoir évaluer la vitesse et l'ampleur de la propagation. Nous pensons au contraire que l'importance de ces phénomènes impose une réévaluation en profondeur des étapes fondamentales que sont l'introduction et l'adaptation d'une nouveauté. Un premier pas dans cette direction consiste à reconnaître que l'analyse des phénomènes de diffusion n'est possible que dans le cadre d'une conception de la culture qui intègre son caractère fondamentalement dynamique. De ce point de vue, le concept de «pratique », défini comme une notion englobant l'usage (de gestes, d'objets, de vocabulaire), mais aussi les comportements, les attitudes et les représentations qui se rapportent à cet usage, est tout à fait fondamental (Bourdieu 1972; Giddens 1979; Pauketat 2001 ; Dobres 2000). La pratique est d'abord un processus par lequel nous pouvons expérimenter le monde d'une manière signifiante, qui nous permet de donner un sens à nos actes. Il en va de même pour les pratiques collectives, dont le sens est collectivement négocié. Considérer les faits de diffusion comme des pratiques parmi d'autres permet de les mettre sur le même pied que tout processus de transformation d'usage. Cela permet également de sortir d'une approche réductrice des diffusions qui se limite à considérer la présence ou l'absence du trait étudié, sans guère manifester d'intérêt pour l'usage qui en est fait.

Les négociations de sens qui se déroulent au moment de l'adoption d'une nouveauté sont non seulement du même ordre que celles qui accompagnent toute transformation des usages, mais elles sont aussi intimement liées aux transformations en cours dans l'espace considéré. Il s'agit de deux dynamiques qui interfèrent, comme le montre très bien Jean Lave (ce volume). En ce qui concerne les bassines, leur adoption ne fait sens que dans le cadre des changements sociaux en cours au Niger.

Considérer les cultures humaines comme fondamentalement dynamiques évite aussi les débats stériles et les affirmations péremptoires, comme celle d'Arnould, qui écrit que les habitants de Zinder « n'ont pas de disposition particulière pour l'innovation » (1989: 240). Il convient en effet de ne pas confondre la course à la distinction sociale à travers l'acquisition de nouveaux objets, qui serait propre à nos sociétés de consommation, et les dispositions à l'innovation, toujours présentes, en attente d'être stimulées par une demande sociale. Au Moyen Âge, par exemple, les teinturiers européens ne savaient pas obtenir un noir profond et durable. Lorsque la mode des vêtements noirs s'est imposée, le problème technique fut résolu en quelques décennies (Pastoureau 1997 : 126). L'histoire des sciences foisonne de récits de ce type - la mise au point des sulfamides et de la pénicilline lors des deux guerres mondiales en sont les exemples les mieux connus. Il en va de même de la petite histoire des objets de la vie quotidienne, comme le Post-it (Petrosky 1992: 80-86). Tous ces cas montrent l'apparition d'une innovation comme la rencontre entre la contingence et la réflexivité. 
nature les éléments dont elles étudient la diffusion. C'est particulièrement explicite chez Rogers (1995). Pourtant, les recherches que nous avons menées montrent qu'il est impossible d'aborder de la même manière des choses aussi différentes qu'une diffusion d'objets, de pratiques culturales (Bastin, ce volume), de techniques de récupération du métal, de vocabulaire technique (Van der Veken, ce volume), ou d'une fête calendaire telle que halloween (Zeebroek, ce volume). Il est donc légitime de s'interroger sur la validité du présupposé selon lequel, en matière de diffusion, tous les « traits culturels» forment une et une seule catégorie. À l'inverse, une approche qui individualiserait à l'extrême les objets et les pratiques serait profondément décevante, car elle s'opposerait à toute tentative de généralisation. Comment alors mettre de l'ordre dans la variété des phénomènes observables?

\section{Participation et réification sont dans un bateau...}

Un premier élément de réponse consiste à souligner que les éléments dont nous étudions la diffusion sont inséparables des groupes humains qui les utilisent. Cela signifie que nous ne pouvons étudier la diffusion de gestes, d'objets ou de vocabulaire comme s'il s'agissait de choses qui ont une existence indépendante des circonstances sociales où elles sont mises en œuvre. Ces éléments sont toujours pris dans une pratique géographiquement, historiquement et socialement située. Il s'agit dès lors d'étudier la trajectoire de « manières de faire » qui mobilisent simultanément des individus, des objets et des représentations (Lave \& Wenger 1991 ; Bonnot 2002; Blandin 2002). Certaines des analyses qu'Étienne Wenger a développées à propos des « communautés de pratique » sont particulièrement éclairantes à cet égard. Dans le cadre de nos activités, écrit-il, nous donnons une forme à notre expérience en produisant des « objets » qui la matérialisent (1998: 58). Ces « objets » sont tout aussi bien les produits matériels d'une activité (des marmites en terre cuite ou en aluminium par exemple) que des mots comme " démocratie », des formules mathématiques, des index du type " pages jaunes », etc. Wenger nomme ces « objets » des réifications.

Qu'il s'agisse d'artefact ou de productions intellectuelles, les réifications incorporent leurs conditions matérielles de production, mais aussi leurs conditions sociales d'usage et les représentations qui y sont associées. C'est ce qu'illustre très bien Jean Lave (ce volume). Les réifications reflètent donc le cadre social dont elles sont issues, mais constituent aussi des points focaux autour desquels la négociation du sens des pratiques ultérieures va s'organiser. En d'autres termes, qu'elles soient produites par la communauté qui les utilise ou qu'elles proviennent d'une autre communauté, les réifications doivent toujours être réappropriées localement pour devenir signifiantes. Prenons l'exemple des règles d'un jeu. Elles 
sont formulées dans le cadre d'une participation des individus à ce jeu participation qui les conduit à en partager une interprétation commune. Lorsque ces règles sont transmises de manière abstraite, sous forme de manuel, sans que la transmission s'accompagne d'une pratique partagée, des divergences d'interprétation émergent alors entre les participants. En d'autres termes, les règles ne transmettent jamais l'ensemble des éléments nécessaires à leur compréhension univoque ou à une "mise en actes » socialement partagée. De même, lorsque le collectif qui met ces règles en pratique évolue, parce que le nombre et la nature de ses participants changent, alors les règles sont susceptibles d'évoluer elles aussi. La diffusion mondiale du judo, par exemple, a contribué à en modifier les règles, en dépit de l'avis des créateurs japonais de ce sport (Rose 2006).

Les réifications acquièrent donc une sorte de vie autonome, qui peut conduire à des interprétations différentes de leur sens, suivant le contexte local où elles sont mises en pratique (Wenger 1998 : 62). Cette dérive du sens dépend de la manière dont la signification est répartie entre la réification et la participation à une pratique qui la met en ouvre: le devenir des objets et des concepts fluctue donc suivant le type d'interaction sociale dans laquelle ils sont pris. Wenger invite ainsi à réfléchir sur « la distance limitée [qu'une réification] peut couvrir dans le temps et dans l'espace, sans accompagnement, en ne courant pas de risques d'interprétations divergentes » (1998: 111). Du point de vue de la diffusion, lorsque des réifications telles que des produits émaillés ou le concept de démocratie sont introduites dans un espace social sans être accompagnées par des individus susceptibles d'en montrer l'usage, alors le sens qui leur est attribué est susceptible de s'écarter fortement de celui qu'elles ont dans leur contexte d'origine. Par exemple, les casseroles exposées au côté des bassines dans les chambres des femmes sont considérées en Afrique de l'Ouest comme des plats de présentation et non des récipients destinés à la cuisson les aliments. Du point de vue africain, c'est notre usage de ces objets qui paraît inapproprié. De même, lorsque les récipients émaillés ont un usage domestique, ils se substituent le plus souvent aux paniers et calebasses de transport, ce qui ne correspond pas non plus à un usage occidental.

Lorsque ce sont des représentations, c'est-à-dire des idées comme celle de « démocratie », qui pénètrent dans des univers où elles sont neuves, elles doivent aussi trouver une place qui leur soit spécifique dans les codes sociaux déjà constitués. Ici encore, la négociation du sens peut entraîner une transformation importante de la pratique associée aux représentations. Un exemple édifiant est fourni par la trajectoire que suit la fête d'halloween en Belgique depuis une dizaine d'années (Zeebroek, ce volume).

Pour que la signification des traits culturels puisse se répartir entre participation et réification, il faut donc nécessairement que des individus soient les vecteurs de ces traits. C'est ici que la nature des relations entre l'individu vecteur et ceux qui appartiennent à l'espace social local devient fondamentale. De celle-ci dépend en effet l'intensité de la participation commune à la nouvelle pratique, laquelle peut être forte, comme dans le cas de la métallurgie de récupération de l'aluminium, où l'on entre seulement par 
apprentissage (Romainville, ce volume), ou faible, comme dans le cas des initiateurs des manifestations d'halloween en Wallonie, qui n'ont qu'une connaissance indirecte et partielle des usages américains (Zeebroek 2006 et ce volume).

La façon dont les « traits culturels » mobilisent des réifications et des participations, et la proportion qu'ils instaurent entre ces deux éléments va donc influencer leur trajectoire et leur signification. Cette proportion détermine le degré de réinvention nécessaire pour que l'adoption mise en pratique dans un contexte situé - et la généralisation de ce trait deviennent effectives. Les caractéristiques du trait ont ainsi des conséquences sur la construction de son utilité sociale, condition indispensable à sa généralisation.

Outre une première mise en ordre dans l'ensemble des traits culturels qui diffusent, cette approche a l'avantage d'articuler les particularités du trait transmis à ses conditions sociales d'introduction dans l'espace considéré.

\section{avantage adaptatif et utilitarisme tombent a l'eau}

Nous avons vu que tout en reconnaissant l'importance des termes de la rencontre avec le système social pour le futur de la nouveauté, Rogers sépare ce phénomène des raisons qui favorisent son adoption. De même, il fait toujours figurer l'avantage relatif des innovations au premier rang des critères qui entraînent la décision d'adoption (Rogers 1995 : chapitre VI). Or, une perspective aussi platement utilitariste est contredite par les études contemporaines consacrées aux techniques et aux objets (par ex., Dobres 2000 ; Lemonnier 1993, 2004; Mahias 2002 ; Petroski 1992; Pfaffenberger 1992; Tilley et al. 2006). Dans le cas des assemblages matrimoniaux au Niger, quels avantages pourraient avoir les bassines sur les calebasses pour qu'elles s'y substituent? Est-ce parce qu'elles rencontrent mieux de nouveaux critères esthétiques (lisse, brillant, polychrome)? Rien n'indique que ces nouveaux goûts soient antérieurs à l'adoption des bassines. Est-ce parce qu'elles permettent de mieux exprimer les différentiations sociales et économiques? Mais de quel type de différenciation parle-t-on, et entre quels groupes? Et pourquoi les bassines plutôt qu'un autre objet? Le même genre de problème se pose lorsqu'on cherche à évaluer les « avantages » qu'apporte l'adoption d'une fête comme halloween. 
Dans l'état actuel de nos recherches, nous ignorons où et quand les bassines émaillées ont changé de statut et ont pénétré dans les chambres des femmes comme objets décoratifs et de prestige. Mais il semblerait que l'usage particulier que nous avons rapporté ne se soit mis en place qu'à partir de la fin des années 1950, vraisemblablement en milieu rural. Cette adoption a été rendue possible par la reprise de la commercialisation de produits industriels européens, puis par la création de structures de production locales, tout particulièrement au Nigeria, au Ghana et en Côte-d'Ivoire. C'est également à cette époque que les villes coloniales deviennent des lieux massivement reconnus par les ruraux comme des endroits où l'on peut se procurer de l'argent. Il s'ensuit une augmentation des migrations entre villes et campagnes et donc de nouvelles possibilités d'approvisionnement en produits manufacturés. Au cours de la même période, on observe une monétarisation progressive de l'économie, associée au développement des cultures commerciales, qui va rendre ces produits plus accessibles. Mais il ne s'agit là que de conditions favorables qui, à elles seules, ne suffisent pas à expliquer le choix des objets émaillés comme bien matrimoniaux.

C'est ici qu'il devient intéressant de faire le détour par les zones « vides », celles où l'innovation n'a pas diffusé. Il est remarquable que les Peuls soient restés fidèles aux calebasses jusqu'aujourd'hui. Pourtant, ils n'ignorent pas les pratiques des villageoises zarma et hausa et parlent de leurs assemblages - ce qui montre, si besoin en était, que la connaissance d'une innovation n'est qu'une condition nécessaire, jamais suffisante pour en assurer la diffusion (contrairement à Rogers 1995). Les Peuls reconnaissent en outre les qualités esthétiques des récipients en métal, particulièrement les inox, comme en témoigne la présence de quelques récipients manufacturés parmi les « trésors » des femmes. Cette incorporation reste pourtant marginale, et les bassines apparaissent surtout dans un cadre utilitaire (par ex., Beckwith \& Van Offelen 1983 : 40-41).

Nous avons vu qu'il faut chercher les raisons de la fidélité aux calebasses, d'une part dans le rapport très personnalisé que les femmes peules entretiennent avec ces objets, et d'autre part dans leur importance symbolique, en tant que «troupeau » des femmes. Plusieurs éléments s'opposent dès lors à la construction d'une homologie entre « bassines émaillées » et « calebasses», qui permettrait de substituer les unes aux autres. Une configuration d'attitudes et de valeurs condamne en quelque sorte les bassines à conserver un statut de récipient utilitaire. Parallèlement, les Touaregs n'accordent aucun intérêt aux bassines dans les trousseaux féminins. Ici ce sont les éléments du couchage, les sacs de voyage et le petit bétail qui sont valorisés (Bernus 1981). Et lorsque des éléments « modernes » apparaissent dans ces trousseaux, il s'agit symptomatiquement de valises Samsonite et de couvertures en acrylique d'origine asiatique.

D'un point de vue méthodologique, ces observations soulignent la nécessité d'étudier de la même manière les zones où la nouveauté est rejetée et celles où elle est adoptée. Une telle approche n'a pas pour but de dresser des cartes de répartition, dont l'ethnographie a bien montré les limites (Bromberger et al. 1983; Voskuil 1983), mais de comprendre en 
profondeur l'influence du contexte sur la construction de l'utilité sociale de la nouveauté. C'est comme cela que Bastin (ce volume) montre que si les paysannes vivant dans la portion la plus excentrée du secteur nigérien de la vallée du Niger n'ont pas adopté la culture maraîchère - au contraire de celles résidant en son cœur -, c'est moins pour des raisons d'éloignement que parce que cette activité y fait l'objet d'une moindre utilité (économique, sociale et culturelle) qu'une série de stratégies concurrentes, comme le commerce du riz, par exemple.

Dans notre cas, la comparaison entre les zones « vides » et « pleines » met en lumière le sens différent que les femmes peules et les femmes hausa accordent aux calebasses. Si les calebasses que recevaient les fiancées hausa étaient décorées par leurs parentes, leur valeur dépendait bien plus de leur nombre que de la qualité de leur décoration. D’une manière générale, dans le monde hausa, la fertilité et la prospérité sont suggérées par la répétition d'objets similaires. Dans le contexte du mariage, il s'agissait aussi de suggérer par le nombre des présents l'étendue des réseaux familiaux et sociaux de la mère de la fiancée. L'étalement de cette richesse en liens sociaux visait surtout à assurer à la fiancée un statut tel qu'elle soit respectée dans la famille de son mari, ce qui, au vu de la structure des maisonnées hausa, n’a rien d'évident (Cooper 1995 : 127-130; Solivetti 1994).

La situation va fortement changer avec la monétarisation de l'économie et le développement du commerce consécutif au « boom » arachidier des années 1950. À partir de ce moment, les femmes s'investissent dans des activités commerciales, et l'argent qu'elles en retirent leur donne accès aux produits industriels, dont les bassines émaillées. Toujours dans la perspective d'asseoir le statut de la fiancée, les bassines sont introduites dans les assemblages «précisément», explique Cooper (1995: 133), " parce que la valeur sociale de la fiancée et de sa mère peut être mieux mise en valeur par des cadeaux qui révèlent l'accès des femmes à un revenu monétaire ». Dans un monde où les revenus monétaires ont acquis plus d'importance que les réseaux de solidarité, les « choses de la chambre » deviennent parallèlement un capital mobile, une sorte d'assurance - par exemple en cas de divorce -, contrairement aux calebasses qui ne pouvaient être vendues (Cooper 1995 : 136).

Enfin, un dernier facteur différencie les Hausa des Peuls et des autres populations qui les entourent. Depuis les années 1950, les prestations matrimoniales hausa ont connu des évolutions marquées par un déplacement du centre de gravité des échanges, anciennement effectués entre les familles des mariés et actuellement orientés vers la constitution du trousseau de l'épousée (Nicolas 1986 : 50-51). L'accent mis sur le trousseau offert à la jeune fille va lui permettre d'exprimer plus clairement ses goûts et ses désirs et donc de mieux se différencier des « affaires des mamans » (Gosselain et al., ce volume; Arnould 1989 : 248).

Lanalyse de cet exemple montre bien que c'est un ensemble d'évolutions agissant à des échelles spatiales et sociales différentes qui a préparé et permis l'intégration des bassines dans les chambres des femmes en tant qu'objet décoratif et de prestige. Il n'y a donc pas de "simple » 
substitution, où une forme plus performante remplacerait une forme ancienne. L'opération témoigne au contraire de la rencontre entre des évolutions internes et les spécificités d'un objet: les bassines sont d'abord des objets concaves, des récipients, et à ce titre sont homologues des calebasses déjà présentes dans les chambres des épouses. Cette ressemblance « ouvre la voie » (Keane 2005: 8) à une mise en relation des objets. Socialement construit, le processus d'adoption ne fait alors qu'étendre le vocabulaire des pratiques, sans en changer la grammaire. C'est ce qui explique que les bassines se soient initialement ajoutées aux collections de calebasses, avant de les remplacer.

C'est bien évidemment l'ensemble des caractéristiques reconnues aux bassines qui explique leur succès. Prise isolément, aucune d'elles n'est suffisante. On pourrait penser, par exemple, que la « modernité » de l'objet est un facteur décisif dans son succès, comme le suggère Platte (2004). Mais comment expliquer alors que les casseroles en aluminium fabriquées au Niger (Romainville, ce volume), elles aussi incontestablement « modernes », ne soient jamais rentrées dans les décorations des chambres? Cette exclusion tient à leur nature d'ustensile de cuisson, ce qui les oppose nettement aux bassines - et même aux casseroles émaillées, qui servent éventuellement de plats de présentation mais jamais d'instruments de cuisson. Tous les récipients servant à cuire les aliments, qu'ils soient en terre cuite ou en aluminium, noircissent immédiatement. Ils ne sont donc que peu investis au plan décoratif, à l'opposé des autres catégories de conteneurs dont l'aspect esthétique est valorisé. Nous comprenons alors pourquoi les casseroles en aluminium n'apparaissent pas dans les « choses de la chambre» malgré leur caractère « moderne » et leur prix relativement élevé.

Le succès des bassines dans le monde hausa est lié à la fois à des transformations socio-économiques et à des tensions intergénérationnelles. Ici, « l'avantage » des objets tient en ce qu'ils permettent aux femmes d'exprimer les nouveaux rapports de différenciation économiques dans un langage socialement partagé, tout en affichant leur « modernité » (sociale et générationnelle) - ce qui a peu à voir avec des critères de performance ou d'avantages, au sens habituel du terme.

L'utilité à laquelle nous sommes confronté est une "utilité sociale », issue d'un processus de construction continu et situé, que l'on peut appréhender dans l'évolution des assemblages au cours du temps et dans leurs variantes régionales. Dire que la construction de l'utilité sociale est un processus continu et situé, signifie qu'à chaque mise en pratique du trait, son utilité est réévaluée par les individus qui le manipulent. Par conséquent, assimiler ce processus toujours recommencé à des phases précises dans une succession linéaire d'étapes (Arnould 1989; Rogers 1995) est terriblement réducteur.

Jean Lave (ce volume) rappelle à quel point il est important, lorsqu'on parle de transmissions culturelles, de ne pas séparer la pensée de l'action. Le découplage entre une phase de perception de la compatibilité du nouveau trait et une phase d'objectivation des avantages apparaît alors pour ce qu'il est: un morcellement analytique bien éloigné du vécu des acteurs. Dans 
notre cas, le trait culturel existe dans l'espace social de référence depuis un certain temps avant son adoption: ce qui change, c'est le regard que les individus portent sur lui. Non pas parce qu'il apparaît soudain comme compatible et avantageux, mais parce qu'il laisse entrevoir une possibilité de réponse nouvelle à la situation du moment.

Plus généralement, on remarquera que les réifications matérielles, comme les bassines, sont particulièrement sujettes à l'intégration par ajout dans une grammaire préexistante avec transformation des pratiques. La même tendance est à l'œuvre dans le cas de réifications immatérielles représentations - comme halloween, dont l'adoption en Wallonie peut être décrite dans les termes d'une intégration à la grammaire du carnaval (Zeebroek, ce volume).

Par contre, les activités productrices, comme la métallurgie de l'aluminium, le maraîchage (Bastin, ce volume) ou l'usage du tour et de la fraiseuse (Stroobants, ce volume) s'inscrivent dans un registre différent. Ici, la transmission de la technique s'effectue par apprentissage - en atelier ou au jardin - ce qui privilégie la participation. Il en résulte une uniformité des pratiques à l'échelle aussi bien régionale (maraîchage) que subcontinentale (métallurgie de l'aluminium). En ce qui concerne la fabrication des marmites en aluminium, d'autres facteurs sont sans doute à l'œuvre, dont nous n'avons pas traité jusqu'ici. Parmi eux, les caractéristiques des espaces sociaux et géographiques dans lesquels les « choses » diffusent. Avec la fonte de l'aluminium, nous sommes confrontés à une activité jeune et techniquement novatrice, qui a vraisemblablement une origine unique (Romainville, ce volume). Sa diffusion s'appuie sur la fabrication de casseroles dont la demande semble actuellement illimitée. Elle s'implante aussi presque exclusivement en milieu urbain. Concrètement, on voit émerger une activité singulière dans un contexte partagé au même moment par bien d'autres villes africaines et caractérisé par un faisceau d'influences exogènes en ce qui concerne la matière première, les outils, le vocabulaire et le cadre professionnel. Lorsque la première génération de fondeurs se déplace pour créer de nouveaux ateliers, elle apporte avec elle non seulement un répertoire de pratiques techniques dont les modalités de transmission par participation favorisent la reproduction, mais elle s'installe également dans des cadres urbains dont les caractéristiques sont globalement comparables à celles de leur point de départ. En d'autres termes, la fonte de l'aluminium est littéralement prise en tenaille entre deux ensembles de déterminations qui concourent à en conserver l'homogénéité originelle.

Ceci ne vaut évidemment que pour la phase initiale d'un processus de diffusion qui s'apparente plus ou moins à un «front pionnier ». Au fil de son expansion spatiale, mais plus encore au fil du temps, la technique est inévitablement prise dans des dynamiques d'évolution et de redéfinitions locales. On relèvera à ce propos l'exemple de ces fondeurs d'origine forgeronne qui maintiennent dans leur nouvelle activité une partie du vocabulaire propre au travail du fer, alors que leurs apprentis d'origine non forgeronne abandonnent ce vocabulaire lorsqu'ils quittent leur patron (Van der Veeken, ce volume). 


\section{Les dimensions spatiales des processus de diffusion}

Comme nous l'avons vu à plusieurs reprises, la diffusion des objets et des pratiques est aussi un processus spatial. Les « lieux du monde » adoptent inégalement et selon des rythmes variés les nouveautés produites ou utilisées ailleurs. Ces différences d'ampleur et de vitesse de propagation intriguent depuis longtemps les géographes. Abordées dès la fin du XIXe siècle par Ratzel, elles ont fait l'objet d'études approfondies depuis les années 1950, suite aux avancées pionnières de Hagerstrand (1957). Comme chez Rogers, ces études envisagent la diffusion sous l'angle de la communication. Elles interprètent l'inégale propagation d'une nouveauté dans l'espace à l'aune des relations qui se tissent entre émetteurs et récepteurs potentiels. Ce faisant, elles mettent en avant le rôle des facteurs susceptibles d'infléchir ces relations, en particulier la distance, la densité de la population, la hiérarchie urbaine et les réseaux de transports (Saint-Julien 1985). Deux types de processus sont ainsi mis en évidence: une diffusion de proche en proche, qui, à l'égal des processus de contagion, serait essentiellement dépendante de la distance entre émetteur et récepteur et une diffusion par la voie de la hiérarchie urbaine, dans laquelle les liens privilégiés entre grandes villes sont perçus comme un facteur clé. On admet aujourd'hui que ces deux formes de diffusion se combinent: si le canal hiérarchique de propagation est souvent prépondérant à petite échelle, il est supplanté par le voisinage à grande échelle.

À ce stade, nous ne disposons pas d'éléments empiriques suffisants pour questionner la dimension spatiale de l'usage matrimonial des bassines émaillées en Afrique de l'Ouest - au-delà du constat d'une distribution très large dans une zone qui pourrait correspondre à l'aire de nomadisation des Peuls (Kesteloot et al. 1993 : 185). Cet exemple n'en permet pas moins de jeter un regard critique sur les travaux que les géographes ont consacré à la question de la diffusion. Tout d'abord, le fait qu'ils envisagent le processus sous l'angle de la communication les conduit, soit à nier le rôle crucial du contexte social et culturel, soit à assimiler celui-ci à un obstacle qui réduirait la vitesse de propagation d'une innovation, voire l'empêcherait localement.

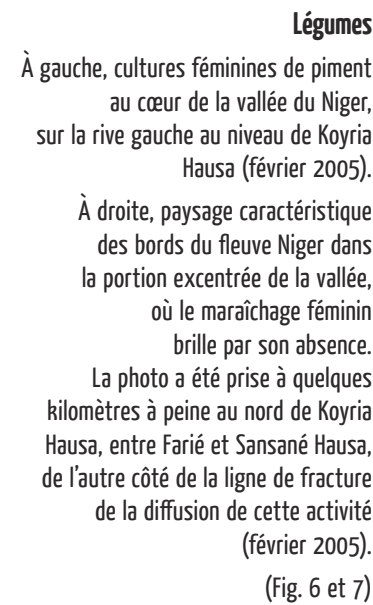

(Fig. 6 et 7 )

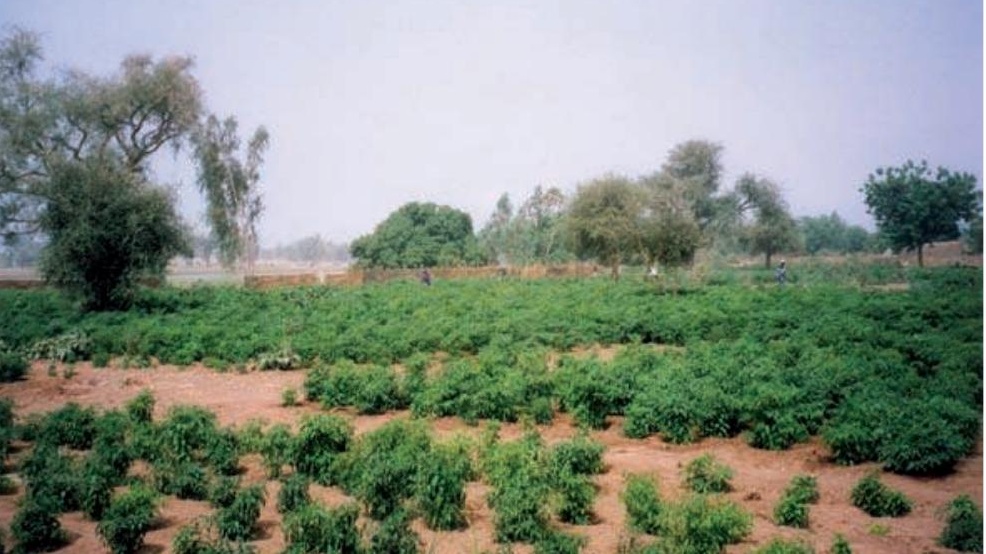




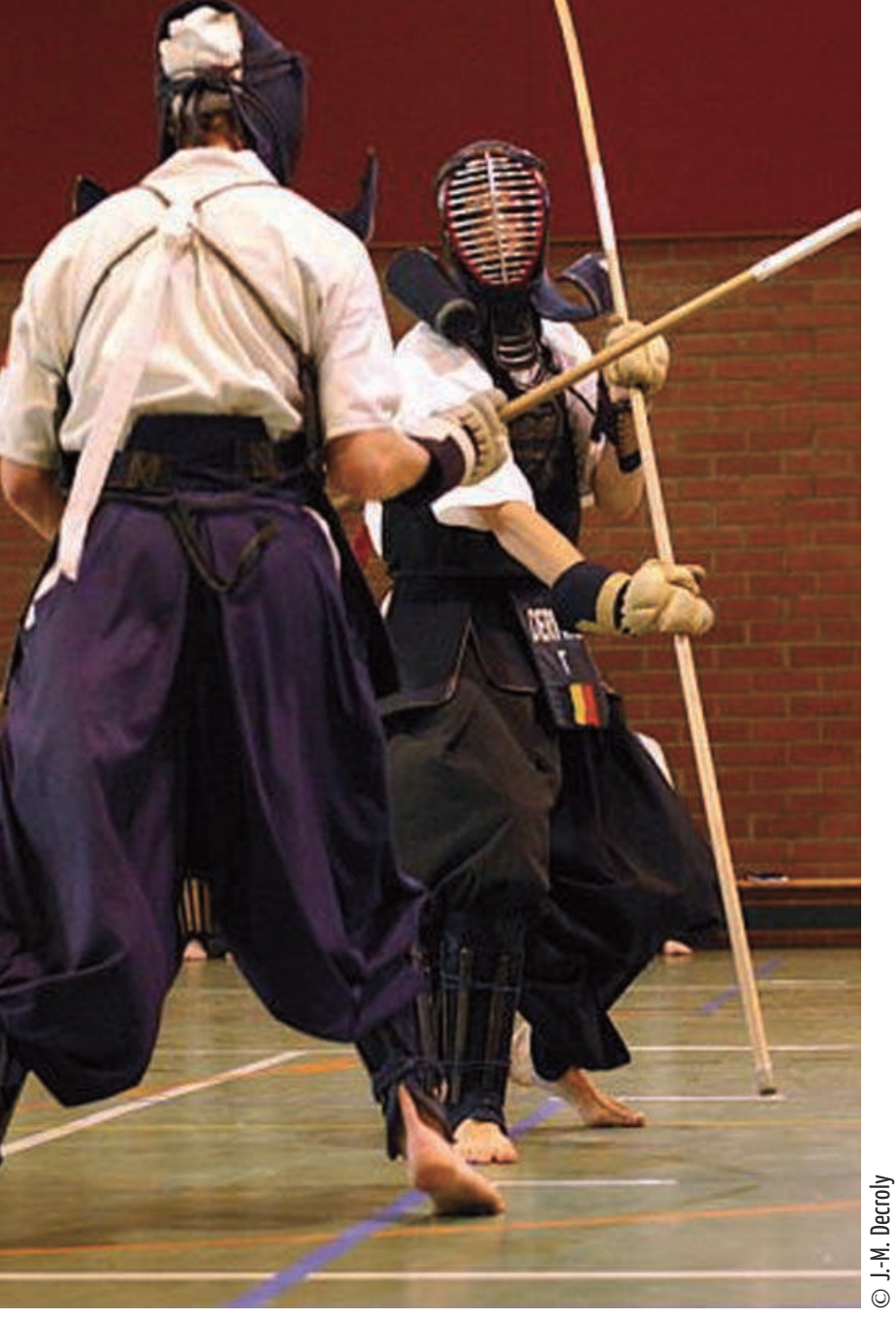

Les travaux menés en géographie ne peuvent donc fournir qu'une interprétation très partielle des processus de diffusion, car ils n'interrogent pas les négociations de sens auxquelles ces diffusions donnent lieu.

En privilégiant les effets de la distance et de la hiérarchie urbaine, ces travaux sous-estiment également l'influence d'autres facteurs, comme les réseaux sociaux, les réseaux commerciaux, les réseaux professionnels et les migrations. L'analyse de ces facteurs se révèle pourtant cruciale, puisqu'elle permet d'identifier précisément le ou les vecteur(s) d'introduction ou d'approvisionnement d'un trait dans l'univers local. Or, des jugements de valeur et des représentations sont associés à ces vecteurs, qui portent tant sur les lieux mis en relation avec l'espace local, que sur les acteurs qu'ils mobilisent et sur la difficulté de leur activation (Gosselain 2008). En ce sens, ils exercent eux-mêmes une influence sur les négociations de sens dont les nouveautés vont faire l'objet.

Au Niger, les modes différenciés d'approvisionnement en bassines et les jugements qui en résultent sont très révélateurs de l'effet exercé par les vecteurs d'introduction sur les modalités de diffusion. Il existe en effet trois sources principales d'approvisionnement en récipients émaillés: les marchés du Niger, les marchés du Nigeria et les marchés des métropoles côtières de Côted'Ivoire, du Ghana et du Togo. Dans les deux premiers

\section{Entrânement au combat de deux naginataka.}

Formalisé en tant qu'art martial à la fin du XIXe siècle au Japon, suite à la création du Budokukaï à Kyoto, le naginata est une escrime qui se pratique pied gauche ou pied droit avancé, avec une lame de bambou fixée à une hampe. II faut attendre le dernier quart du XXe siècle pour qu'elle se développe en dehors du Japon. Dans la trajectoire de cet art martial, l'apprentissage par participation laisse peu de place aux réappropriations locales.

(Fig. 8) cas, les produits émaillés sont achetés dans le cadre de déplacements « pour achat », généralement brefs et courts. Dans le troisième cas, les produits sont acquis dans le cadre de migrations circulaires, longues de plusieurs mois et motivées par la recherche de revenus complémentaires. Plus que le lieu de fabrication lui-même, ce sont les modalités d'acquisition des bassines qui établissent la hiérarchie de leur valeur. De manière générale, les produits acquis au Niger ou au Nigeria sont méconsidérées, surtout en milieu urbain. Disponibles en grand nombre, ils s'obtiennent facilement, même au Niger, et en particulier dans l'aire hausa, où la contrebande transfrontalière est quasi institutionnelle (Grégoire 1986). Quant aux produits en provenance des villes côtières, ils sont considérés comme plus prestigieux, non seulement en raison de leur origine et de leur rareté relative, mais surtout parce qu'ils sont investis de représentations positives associées aux migrations circulaires et à leurs destinations urbaines.

L'exemple des produits émaillés indique également que la relation entre mondes urbain et rural est loin de présenter le caractère simple et univoque que lui prêtent ceux qui considèrent que les impulsions aux changements viennent exclusivement des villes. Selon les éléments disponibles, la substitution des calebasses par des bassines se serait d'abord déroulée dans les campagnes, suite à la monétarisation de l'économie. Ce n'est que dans 
un second temps, avec l'émergence d'une élite urbaine de salariés, que les bassines auraient fait leur apparition dans le trousseau de mariage des citadines. Enfin, la croissance de la population urbaine et l'affirmation du statut privilégié de cette dernière ont contribué à transformer les villes en lieux d'impulsion. Suivant une logique de distinction classique, l'usage d'étagères pour disposer l'assemblage a d'abord émergé en ville, pour se propager ensuite dans le monde rural. De la même manière, l'abandon récent des produits émaillés au profit de bibelots en porcelaine ou en verre n'a jusqu'à présent été observé qu'en milieu urbain. Par ailleurs, les relations dialectiques entre la ville et la campagne illustrent à nouveau le rôle des réseaux (familiaux, commerciaux et professionnels) et des migrations dans les processus de diffusion. Par exemple, les armoires vitrées ont été introduites en milieu rural par des jeunes filles ayant des connexions urbaines ou par des menuisiers formés dans les grandes villes qui ont ouvert des ateliers dans des localités de moindre importance.

L'inscription spatiale des réseaux familiaux, commerciaux et professionnels constitue donc bien une clef de lecture essentielle des processus de propagation de nouveaux objets ou de nouvelles pratiques. On se gardera toutefois de surestimer son pouvoir explicatif. D'une part, avec l'extension sans précédent des moyens de communication de masse, des réifications comme halloween peuvent être introduites dans un espace social sans passer par un quelconque réseau autre que la radio, la télévision ou la presse (Zeebroek, ce volume). D'autre part, l'existence d'une relation familiale, commerciale ou professionnelle entre deux espaces sociaux ne fait pas automatiquement de celle-ci le vecteur d'une diffusion. Ainsi, les relations commerciales entretenues par les Peuls Wodaabe avec les communautés rurales leur permettent de connaître l'usage ornemental des produits émaillés chez les Zarma et les Hausa, ce qui ne conduit pas pour autant à une adoption. De même, une analyse comparée des traditions céramiques dans la région du fleuve Niger montre que le statut social des potières constitue un filtre puissant dans la circulation des façons de faire. Certaines femmes connaissent et estiment une catégorie particulière de décor ou de technique de façonnage, mais ne l'adoptent pas, craignant d'être assimilées au statut inférieur de leurs productrices (Gosselain 2008).

\section{Pour une approche multiscalaire des processus de diffusion}

Au terme de cette analyse, il paraît évident que l'adoption, la transformation ou le rejet éventuel d'une nouveauté dans un espace social donné dépend à la fois des caractéristiques propres à la nouveauté, des particularités des vecteurs d'introduction et de celles du contexte local dans ses dimensions historiques, économiques et culturelles. Les modalités et les effets d'une diffusion au sein d'un espace donné sont donc forcément singuliers. 


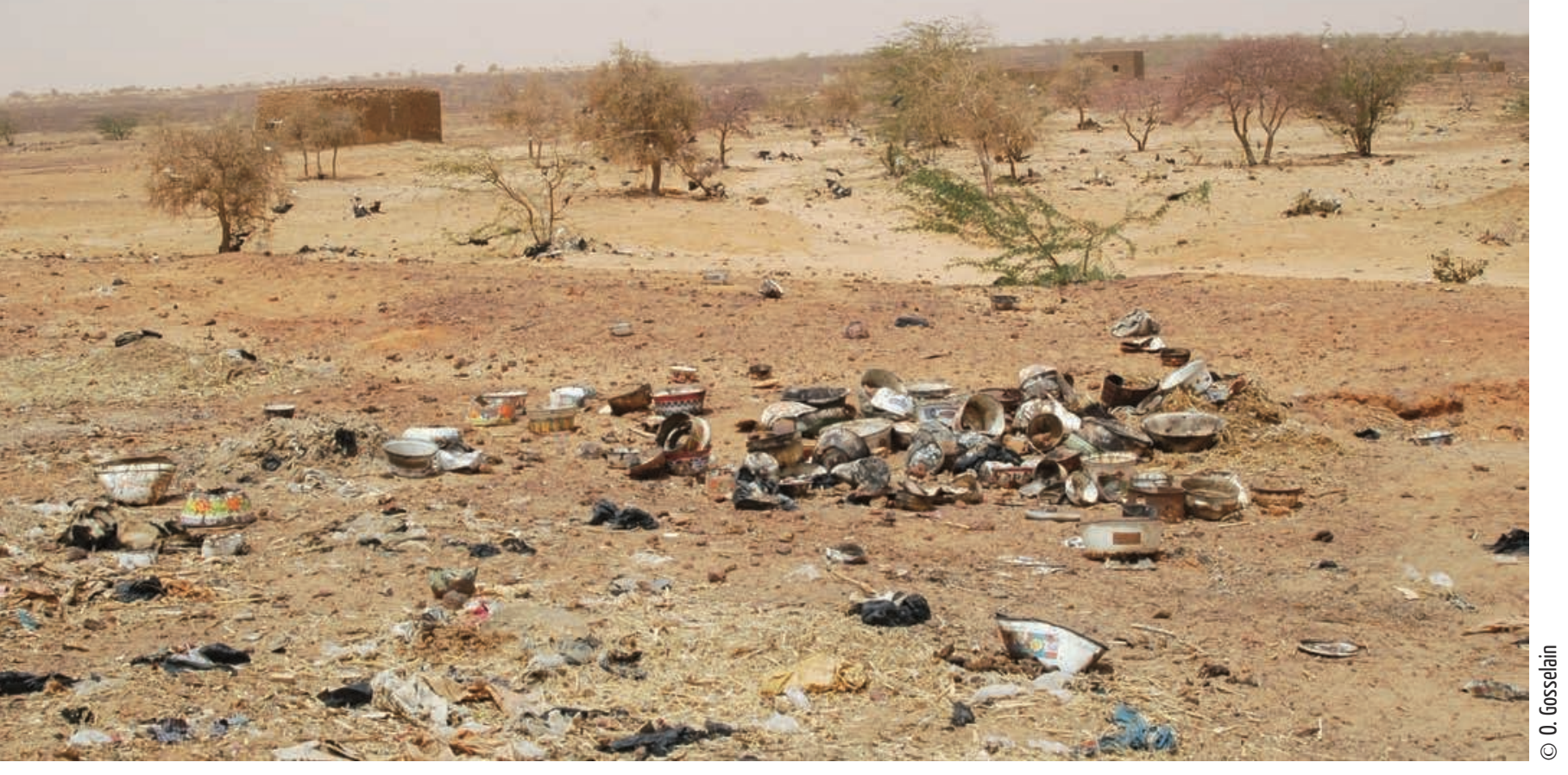

\section{Récipients émaillés à l'abandon}

En Afrique de l'Ouest, la trajectoire de vie d'une bassine peut être longue et partiellement liée à celle de ses utilisatrices. Conçue comme un objet utilitaire, elle entre souvent en scène au moment du mariage, pour passer ensuite de main en main à travers le réseau de parenté et d’amitié. Démodée et ébréchée, elle transite de la sphère des biens de prestiges à celle des biens d'usage courant, finissant sa vie sur les « fumiers », lorsque son état ne permet plus aucun usage.

(Fig. 9)
Comment ne pas céder alors à la tentation relativiste? Il nous semble que la meilleure stratégie consiste à analyser les dynamiques culturelles observées dans une communauté en tant que combinaison de processus qui se déploient à différentes échelles spatiales et selon des logiques propres à chacune d'elles. Une telle approche permet d'identifier à la fois les facteurs qui pèsent, à chaque niveau, sur la diffusion d'objets ou de pratiques, et la manière dont ces facteurs « monoscalaires » s'articulent avec des facteurs agissant à d'autres échelles.

Comme nous l'avons vu, l'usage matrimonial des produits émaillés témoigne d'un emboîtement de processus qui s'inscrivent dans des échelles spatiales et temporelles différentes. Rappelons-en rapidement les aspects les plus saillants. Au niveau global, la diffusion des bassines trouve son origine dans l'expansion européenne, qui a notamment entraîné une exportation massive de récipients manufacturés - produits émaillés, mais également faïences, grès, bouteilles, etc. - en Afrique comme dans d'autres parties du monde (Argenti 1999; Cornevin 1981; Hoskins 1998; Marshall \& Maas 1997; Thomas 1991). La géographie des contacts avec l'occident fournit donc un premier filtre spatial pour décrypter le processus de diffusion.

Au niveau du continent africain, les zones dans lesquelles ces récipients sont apparus correspondent aux lieux où existait un contact effectif entre colons et populations autochtones: comptoirs littoraux, postes administratifs et militaires et arrière-pays des uns et des autres. Les habitants de ces régions ont amorcé l'appropriation de ces différents produits, à la fois comme objets de prestige et comme moyens d'agir sur un monde en profonde mutation (Argenti 1999; Masquelier 2001; Rowlands 1996). 
À un niveau plus régional, ces logiques générales d'appropriation se déclinent selon des modalités différentes, en fonction des contextes culturels. Certains objets, dont la saillance est culturellement reconnue, deviennent le support d'un investissement spécifique. C'est le cas des bassines émaillées en Afrique occidentale, qui sont largement représentées dans la sphère fonctionnelle depuis l'entre-deux-guerres.

À un quatrième niveau, se dessine un sous-espace dans lequel les bassines émaillées ont été insérées dans les trousseaux de mariage. La zone où se manifeste cette grammaire singulière correspond grosso modo à l'aire de nomadisation des populations de langue peule (Gosselain et al., ce volume), chez qui les assemblages matrimoniaux comprennent prioritairement ou exclusivement des calebasses décorées. Cette pratique pourrait avoir servi d'exemple aux populations avoisinantes, à l'exception des Touaregs qui exploitent d'autres catégories d'objets pour exprimer la richesse des femmes, ce qui explique l'existence d'une frontière nette vers le nord.

Dans l'espace nigérien, cinquième niveau, les populations qui ont adopté les bassines émaillées utilisaient précédemment des calebasses et ont opéré une substitution qui n'est pas attestée chez les Peuls Wodaabe. En outre, les différentes communautés témoignent d'un certain nombre de variantes dans l'usage ornemental des produits émaillés. Il semblerait, par exemple, que l'affichage de la richesse constitue la motivation principale de la constitution des trousseaux chez les Hausa, tandis que la matérialisation de l'identité prévaut dans la zone du fleuve (Gosselain et al., ce volume).

L'emboîtement de ces cinq niveaux est en outre traversé par des oppositions entre les zones urbaines et rurales, espaces ou les évolutions des assemblages se produisent à des rythmes différents. Ce dernier facteur conduira vraisemblablement à l'élimination de l'usage des produits émaillés en contexte matrimonial.

\section{$\&$}

Au final, les facteurs mis en évidence pour chacune des échelles considérées s'articulent, se renforcent ou s'inhibent pour donner naissance aux distributions singulières que nous observons. Parmi ces facteurs, certains agissent simultanément à plusieurs échelles et ont donc un rôle moteur dans les différentes manières dont les individus se saisissent du trait observé et l'insèrent dans leur répertoire de pratiques. On peut alors en revenir à la proposition de Keane (2005) au sujet de ces conditions qui « ouvrent la voie » aux processus de mise en relation entre gestes, objets, lexiques et individus. Toutefois, si cette « ouverture » relève bien d'une « offre », nous devons impérativement en repenser la nature. En effet, il ne s'agit pas de l'introduction d'une nouveauté, pourvue d'une ou plusieurs qualité(s) intrinsèque(s) visant à répondre à un besoin précis, mais d'un processus de construction continu, au cours duquel les multiples aspects 
d'un objet ou d'une pratique - le « paquet de potentialités »-sont mis en relation, à différentes échelles, avec la trajectoire biographique des individus. C'est en ce sens que les phénomènes de diffusion sont inévitablement dynamiques et situés.

\section{Sacs de choux en vente au Niger}

La propagation du maraîchage féminin ne relève pas uniquement de la transmission d'un savoir-faire technique, mais également de l'adoption de nouvelles pratiques culinaires et de nouvelles saveurs. Légume inconnu au Niger, le chou a partiellement trouvé sa place comme substitut des feuilles de Moringa oleifera dans la confection du « kopto », un met très apprécié au sud du Niger.

(Fig. 10)

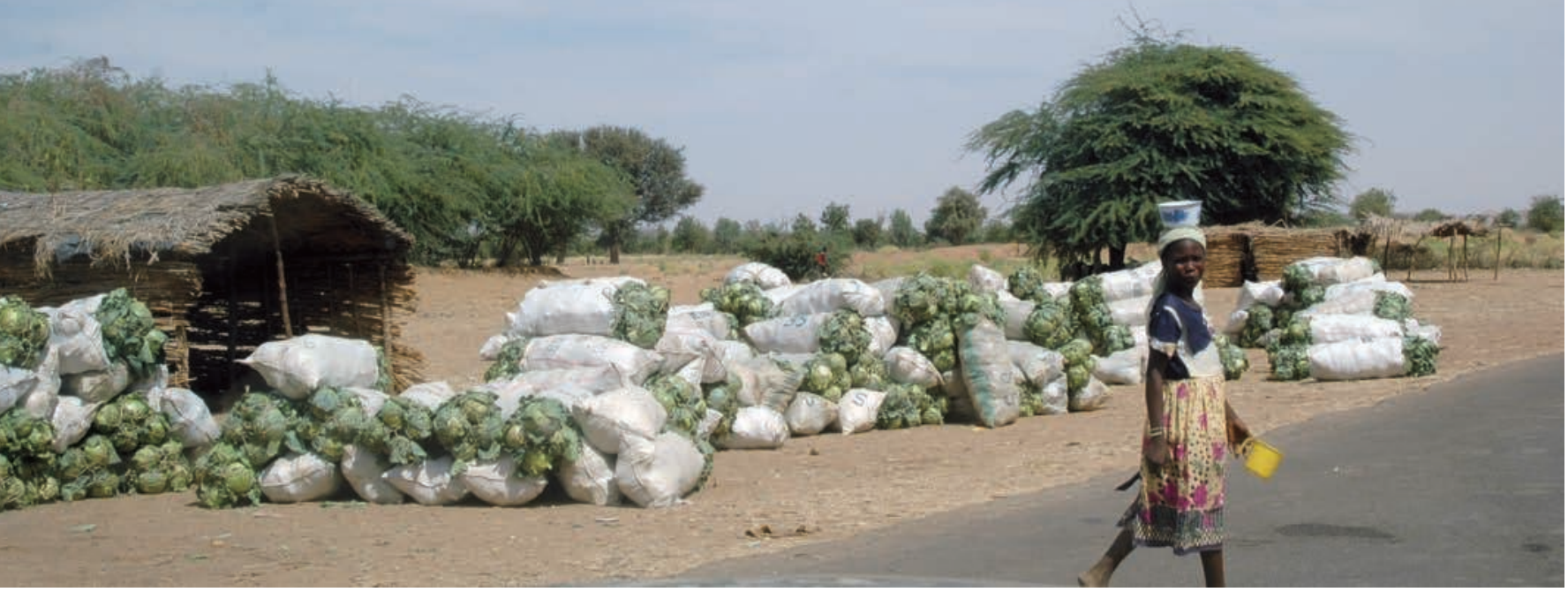




\section{RÉFÉRENCES}

Appadurai, Arjun ed., 1986, The Social Life of Things. Commodities in Cultural Perspective. New York, Cambridge University Press.

Argenti, Nicolas, 1999, Is This How I Looked When I First Got Here? Pottery and Practice in the Cameroon Grassfields. London, British Museum Occasional Paper 132.

Arnould, Eric J., 1989, "Toward a Broadened Theory of Preference Formation and the Diffusion of Innovations: Cases from Zinder Province », Niger Republic. Journal of Consumer Research 16: 239267.

Beckwith, C. \& M. Van Offelen, 1983, Nomads of Niger. New york, Abradale Books

Bernus, Edmond, 1981, Touaregs nigériens. Unité culturelle et diversité régionale d’un peuple pasteur. Paris, L'Harmattan.

Blandin, Bernard, 2002, La Construction du social par les objets. Paris, Presses Universitaires de France.

Bonnot, Thierry, 2002, La Vie des objets : d'ustensiles banals à objets de collection. Paris, Maison des Sciences de l'Homme.

Bourdieu, Pierre, 1972, Esquisse d’une théorie de la pratique. Précédé de trois études d'ethnologie kabyle. Paris, Droz.

Bromberger, Christian, D. Dessotto \& T.-K. Shippers, 1983, «L'ethnocartographie en Europe: coups d'oeil rétrospectifs et questions ouvertes », Technologies, Idéologies, Pratiques 4 (1-4): 15-33.

Cooper, Barbara 1995, «Women's Worth and Wedding Gift Exchange in Maradi, Niger, 1907-1989 », The Journal of African History 36 (1): 121-140.

Cornevin, Robert, 1981, La République Populaire du Bénin. Des origines dahoméennes à nos jours. Paris, Maisonneuve \& Larose.

Robert Cresswell, 1983, «Transferts de techniques et chaînes opératoires », Techniques \& Culture 2: 143163.

Dobres, Marcia-Anne, 2000, Technology and Social Agency. Outlining a Practice Framework for Archaeology. Oxford, Blackwell.

Edgerton, David, 1998, « De l'innovation aux usages: dix thèses éclectiques sur l'histoire des techniques », Annales, Histoire, Sciences Sociales 53 (4-5): 815-837.

Gardi, R., 1974, Maisons africaines. L'art traditionnel de bâtir en Afrique occidentale. Paris, Elsevier Séquoia.

Gatignon, Hubert A. \& Thomas S. Robertson, 1985, « A Propositional Inventory for New Diffusion Research », Journal of Consumer Research 11: 849-867.

Giddens, Anthony, 1979, Central Problems in Social Theory: Action, Structure and Contradiction in Social Analysis. London, Macmillan.

Gosselain, Olivier P., 2008, « Mother Bella was not a Bella. Inherited and Transformed Traditions in Southwestern Niger », In Miriam Stark, Brenda Bowser \& Lee Horne eds, Cultural Transmission and Material Culture: Breaking Down Boundaries. Tucson, The University of Arizona Press: 150-177.

Grégoire, Emannuel, 1986, Les Alhazai de Maradi (Niger). Histoire d'un groupe de riches marchands sahéliens. Paris, Éditions de l'ORSTOM.

Hägerstrand, Torsten, 1957, Innovation Diffusion as a Spatial Process. Chicago, University of Chicago Press. Hoskins, Janet, 1998, Biographical Objects. How Things Tell the Stories of People's Lives. New York, Routledge.

Keane, Webb, 2005, "The Hazards of New Clothes: What Signs Make Possible », in Susan Küchler \& Graeme Were eds, The Art of Clothing. A Pacific Experience. London, UCL Press.

Kesteloot, L., C. Barbey \& S.M. Ndongo, 1993, « Les Peul », in J. Devisse ed., Vallées du Niger. Paris, Réunion des Musées Nationaux: 173-189.

Lave, Jean \& Étienne Wenger, 1991, Situated Learning: Legitimate Peripheral Participation. Cambridge - New York, Cambridge University Press.

Lemonnier, Pierre, 1993, Technological Choices : Transformation in Material Culture since the Neotithic. London, Routledge. 
Lemonnier, Pierre, 2004, « Mythiques chaînes opératoires », Techniques \& Culture 43-44: 25-43.

Leroi-Gourhan, 1992 [1945], Milieu et techniques. Paris, Albin Michel (Sciences d'Aujourd'hui).

Mahias, Marie-Claude, 2002, Le Barattage du monde. Essai d'anthropologie des techniques en Inde. Paris, Éditions de la Maison des sciences de l'homme.

Marshall, Yvonne \& Alexandra Maas, 1997, « Dashing Dishes », World Archaeology 28 (3) : 275-290.

Masquelier, Adeline, 2001, Prayer has Spoiled Everything. Possession, Power, and Identity in an Islamic Town of Niger. Durham, Duke University Press.

Miller, D. ed., 1995, Acknowledging Consumption. London, Routledge.

Miller, Daniel, 1997, «Why Some Things Matter », in D. Miller ed., Material Cultures. London, UCL Press: 3-21.

Nicolas, Guy, 1986, Don rituel et échange marchand dans une société sahélienne. Paris, Institut d'Ethnologie.

Pastoureau, Michel, 1997, Jésus chez le teinturier. Couleurs et teintures dans l'Occident médiéval. Paris, Éditions du Léopard d'Or.

Pauketat, R., 2001, « Practice and History in Archaeology. An Emerging Paradigm », Anthropological Theory $1(1): 73-98$.

Petroski, Henry, 1992, The Evolution of Useful Things. New York, Alfred A. Knopf.

Pfaffenberger, Bryan, 1992, « Social Anthropology of Technology », Annual Review of Anthropology 21 : 491516.

Platte, Edita, 2004, « Towards an African Modernity: Plastic Pots and Enamel Ware in Kanuri-Women's Rooms (Northern Nigeria)», Paideuma 50: 173-192.

Roberts, J. R., C. C. Moore \& A. K. Romney, 1995, « Predicting Similarity in Material Culture Among New Guinea Villages from Propinquity and Langage », Current Anthropology 36: 769-788.

Rogers, Everett, 1995 (4th ed.), Diffusion of Innovations. New-York, The Free Press.

Rose, Charles, 2006, La Diffusion spatiale des arts martiaux en Belgique francophone. Mémoire de licence en sciences géographiques, Université Libre de Bruxelles.

Rowlands, Michael, 1996, « The Consumption of an African Modernity », in Mary Jo Arnoldi, Christraud Geary \& Kris L. Hardin eds, African Material Culture. Bloomington, Indiana University Press: 188213

Saint-Julien, Thérèse, 1985, La Diffusion spatiale des innovations. Montpellier: GIP RECLUS (Collection Reclus modes d'emploi).

Solivetti, Luigi M., 1994, « Marriage and Divorce in a Hausa Community. A Sociological Model », Africa 64 (2): 252-271.

Thomas, Nicholas, 1991, Entangled Objects. Exchange, Material Culture and Colonialism in the Pacific. Harvard University Press.

Tilley, Christopher, Webb Keane, Susanne Küchler, Mike Rowlands \& Patricia Spyer eds, 2006, Handbook of Material Culture. London, Sage Publications.

Voskuil, Johannes J., 1983, « Les limites de la méthode cartographique », Technologies, idéologies, pratiques 4 (1-4) : 105-116.

Wenger, Etienne, 1998, Communities of Practice. Learning, Meaning, and Identity. Cambridge, Cambridge University Press.

Zeebroek, Renaud, 2006, « Persistance ou transformation? La trajectoire d'une fête », Ethnologie Française 36 (2): $321-331$. 


\section{RÉSUMÉ}

Casseroles, légumes et halloween. Une approche multiscalaire des phénomènes de diffusion. Revenant sur la trajectoire des produits émaillés au Niger (Gosselain et al., ce volume) et sur les autres études de cas présentées dans ce Thema, nous proposons un cadre d'analyse général des phénomènes de diffusion. L'article s'ouvre sur une critique de la théorie de « diffusion des innovations » développée par Rogers. Fondamentalement utilitariste, cette théorie se focalise sur les qualités intrinsèques des innovations et sur les conditions de leur propagation, laissant dans l'ombre les moments clés qui vont de l'introduction d'une nouveauté à son adoption. Pour comprendre cette trajectoire complète et les transformations qui l'accompagnent, il s'agit à la fois de reconnaître le caractère fondamentalement dynamique des cultures humaines et de considérer les faits de diffusion comme des pratiques parmi d'autres, qu'il faut analyser parallèlement aux évolutions en cours dans un espace considéré.

Pris dans des pratiques situées, les éléments qui diffusent doivent être abordés comme des « manières de faire » qui mobilisent simultanément des individus, des objets et des représentations. Certains concepts issus de l'analyse des « communautés de pratique » sont particulièrement éclairants à cet égard, car ils nous invitent à considérer la manière dont la signification est répartie entre des objets - matériels ou intellectuels - et les pratiques qui les mettent en œuvre. Évaluer la façon dont les « traits culturels » qui diffusent mobilisent ces deux aspects, permet de mieux comprendre les spécificités de leur trajectoire et leurs éventuelles transformations de sens.

Au final, l'adoption, la transformation ou le rejet d'une nouveauté dans un espace social donné paraît dépendre à la fois des caractéristiques propres à la nouveauté, des particularités des vecteurs d'introduction et de celles du contexte. Pour donner du sens à cet ensemble de déterminations, chaque fois unique, il faut envisager les dynamiques culturelles en tant que combinaison de processus qui se déploient à différentes échelles spatiales et suivant des logiques qui leur sont propres. C'est la manière dont ces différents facteurs s'articulent, se renforcent où s'inhibent qui donne naissance aux distributions singulières que nous observons.

\section{ABSTRACT}

Multiscalar Approachs of Diffusion Phenomena. Reconsidering the trajectory of enamelware in Niger (Gosselain et al., this volume), as well as the other case studies included in this "thema", our aim is to develop a general framework for analyzing diffusion processes. The paper starts with a critique of Rogers' theory of the "diffusion of innovation". Mainly utilitarian in nature, this theory focuses on the intrinsic qualities of innovations and on the conditions under which such innovations propagate, pushing into the background crucial stages of the process, from the introduction of a new element up to its adoption. In order to understand the complete diffusion process and its associated transformations, one must both acknowledge the dynamic character of human cultures and consider diffusion as a practice among others, that must be analysed in connection with ongoing changes in the area under study.

Embedded in situated practices, diffusing elements should be approached as "ways of doing" that simultaneously mobilize individuals, objects, and representations. Some concepts developed in the analysis of "communities of practice" are particularly enlightening in that regard. They compel us to consider the way in which meaning is distributed between things - both material and immaterial - and the practices that implement them. In evaluating the way in which diffusing "cultural traits" draw on these two aspects, one gains a better understanding of the characteristics of their trajectories and possible transformations of meaning.

All in all, the adoption, transformation, or rejection of a new element in a given social space seems to depend on the characteristics of the element considered, the characteristics of the vectors through which it was introduced, and the characteristics of the context within which it is introduced. In order to make sense of such irreducibly unique combination of determining factors, cultural dynamics have to be approached as a combination of processes that take place at various geographical scales and according to their own logics. It is the specific way in which these different factors combine or inhibit themselves that gives rise to the particular distributions observed.

\section{MOTS CLÉS}

Diffusion, pratique située, réification, participation, échelle spatiale.

\section{KEYWORDS}

Diffusion, situated practice, reification, participation, spatial scale. 